\title{
ARQUITECTURA COMO DANZA. DELEUZE, EL ESPACIO Y LAS LÍNEAS DE FUGA
}

\author{
Architecture as Dance. Deleuze, Space and Lines of Flight \\ Andrea Potestá \\ Pontificia Universidad Católica de Chile, Santiago, Chile \\ apotesta@uc.cl
}

\section{Resumen}

A partir de una valoración del gesto corpóreo y del movimiento, el artículo pretende reflexionar sobre el problema de la creación del espacio en arquitectura, subrayando su carácter dinámico y marcando así un contrapunto con la visión tradicional que, desde Hegel, asocia la arquitectura a la mera elaboración de una espacialidad monolítica o estática. Se pretende de este modo establecer una analogía entre la construcción arquitectónica, esto es, la práctica de edificación de muros y divisiones espaciales, y la creación de espacialidad propia de la danza. Siguiendo unas intuiciones de Wittgenstein y de Lyotard y, sobre todo, enfatizando la apuesta transformadora de la noción deleuziana de "línea de fuga", se quiere avanzar la hipótesis de que es posible concebir una profunda continuidad entre la dinámica del cuerpo danzante y la dinámica del cuerpo fluyente a través de los espacios.

Palabras clave: Deleuze, arquitectura, danza, movimiento, líneas de fuga.

\begin{abstract}
Based on an evaluation of the corporeal gesture and movement, the article aims to reflect on the problem of the creation of space in architecture, underlining its dynamic nature and thus marking a counterpoint to the traditional vision that, from Hegel, associates architecture with the simple mere elaboration of a monolithic or static spatiality. The aim is to establish an analogy between architectural construction, that is, the practice of building walls and spatial divisions, and the creation of dance's own spatiality. Following some general intuitions of Wittgenstein and Lyotard and, above all, emphasizing the transformative stake of the Deleuzian notion of "line of flight", an hypothesis is proposed: that it is possible to conceive a deep continuity between the dynamics of the dancing body and the dynamics of the flowing body through spaces.
\end{abstract}

Keywords: Deleuze, architecture, dance, movement, lines of flight.

Fecha de Recepción: 01/07/2019 - Fecha de Aceptación: 26/11/2019 
¿Hacia dónde nos movemos nosotros? [...] ¿No nos precipitamos permanentemente? ¿Y también hacia atrás,
hacia adelante, hacia todos los lados? ¿Hay aún un arriba y un abajo? ¿No erramos como a través de una nada infinita? ¿No sentimos el hálito del espacio vacío?

Friedrich Nietzsche (802)

El movimiento en arquitectura es el acto común del edificio y del caminante.

Benoît Goetz (2011 144)

\section{Gesto arquitectural}

"Un edificio es un gesto", observaba Wittgenstein en un aforismo de 1942, y agregaba lo siguiente: “Un hombre será encarcelado en una habitación que no tiene llave y cuya puerta se abre hacia adentro, si no se le ocurre tirar de ella en vez de empujarla" (Wittgenstein 92 [trad. mod.]). El ejemplo de Wittgenstein es elocuente: la dimensión del gesto (el tirar o el empujar la puerta) determina la misma naturaleza de un edificio (una cárcel o una habitación cualquiera), ya que el cuerpo se dispone en el espacio habitándolo, territorializándolo, esto es, definiendo sus posibilidades de movimiento. Una casa, por ejemplo, no puede separarse sino con gran esfuerzo abstractivo de las maneras de estar en el espacio que ella vuelve posibles. El gesto se sitúa en ese sentido entre lo sólido y el flujo, entre la estructura rígida del edificio y las fuerzas y los dinamismos que acontecen en él. Punto de tránsito y de circulación del sentido del movimiento, el cuerpo determina, en rigor, la propia espacialidad (o espaciosidad) del espacio.

De manera análoga a Wittgenstein, Jean-François Lyotard hablaba de la casa a la luz de la frecuentación y del habitus. En Miseria de la filosofía, decía lo siguiente:

Tener un cuchillo en un cajón de la cocina al alcance de la mano, un libro abierto al lado de la cama, [...] significa estar en fase con su propia rítmica corpórea. [...] A las cuatro de la noche, bajo la escalera, atravieso el piso evitando los muebles, pasando por las puertas, y voy al refrigerador para tomar un vaso de leche - sin encender la luz. Allí donde puedo ser somnámbulo sin errores, allí está mi casa (Lyotard 200).

En el presente artículo se pretende cuestionar el fondo problemático de la conexión de cuerpo y espacio asumida por Wittgenstein y Lyotard y enfatizar los 'gestos arquitecturales', esto es, aquello gestos que abren el espacio para su uso corpóreo, insistiendo o, de ser posible, radicalizando la intuición anunciada en esas citas. En este sentido, se trata de comprender la correlación de cuerpo y espacio, pero sin caer en la simplificación de considerarla como una mera correspondencia 'ergonómica': pensarla de esta forma equivaldría a subrayar la economía y la funcionalidad del espacio, es decir, su sumisión al uso, y, de este modo, renunciar a cuestionar el fondo genético de la espacialidad en tanto tal. En otras palabras, se trata aquí de mostrar que la espacialidad en su conjunto vuelve posible el gesto en la 
misma medida en que el gesto vuelve posible la espacialidad, y que solo a la luz de esta reciprocidad o de este quiasmo primitivo la arquitectura ha podido en el último siglo reinventarse y repensarse (y pensarse, en lo específico, como una proyección o una variación de la danza).

\section{Evadir el espacio}

Es cierto, de hecho, que empezar por el gesto modifica profundamente el enfoque acerca del espacio. Tal como escenarios completamente transformados se abren cuando la filosofía se mueve desde el cuerpo anatómico-utilitario hasta el cuerpo desbordado, performativo, pensado como una epifanía de fuerzas que exceden toda posible reunificación, así, del mismo modo, escenarios inéditos se describen cuando se piensa al espacio como "cosmogénesis del movimiento". Autores como Artaud, Merleau-Ponty, Simondon, Deleuze y Guattari, no hicieron sino desactivar el Sujeto como punto de retorno, como ese punto geométrico sin extensión que la metafísica había ido construyendo, para poner en su lugar las varias producciones de subjetividad que cortan el flujo y los planos múltiples de naturaleza social, política, estética. Con ellos, el pensamiento se ha instalado fuera de la conciencia, en un mundo de conjunciones y de encuentros, de relaciones y de fuerzas, cada vez singulares e imprevisibles.

Ahora bien, ¿qué pasa análogamente con la arquitectura? ¿Qué otro pensamiento del espacio se posibilita? Tradicionalmente, la arquitectura se ha definido como instalación de inmovilidad; se ha asumido que se logra 'algo arquitectural' en la medida en que se logra una estructura estable, cuando se estabiliza una unidad espacial que no se disuelve (que no se hace pedazos). La arquitectura -el proyecto de una organización espacial- mantiene unido lo que, sin ella, no se sostendría de pie. Con este preámbulo somos llevados a pensar la arquitectura como un arte del mantenimiento de los espacios, del estancamiento de los lugares, y no del movimiento o del gesto. Un edificio, por humilde y común que sea, habrá ya, de partida, sido definido por una espacialidad de la inercia: unos perros marcarán todos los días esa esquina, unos letreros serán instalados por un costado de la casa para mantener igual el sentido de la marcha y un habitus se habrá establecido para todos aquellos que circulan en y afuera de él. El edificio está ahí, abierto al tiempo, pero con una usura lenta, llevando su aire de época tal vez, pero manteniendo el espacio fijo e inerte: los objetos arquitectónicos son una cuña de tiempo que se acuña en el tiempo, una cuña de espacio que se acuña en el espacio. Son sólidos, plenos, dureza fija, cristalización de la materia, alrededor de la cual se describen movimientos, pero no siendo ellos mismos en ningún sentido constituidos por la movilidad. 
Es desde este ángulo que se ha entendido la arquitectura generalmente en el sistema de las artes y, no por casualidad, desde Hegel a lo menos se clasifica a la arquitectura en una escala mínima de valores espirituales (cf. Hegel 21). Pintura, música y poesía quedarían encima suyo por haber sabido ir más allá de la materialidad extensiva y haber permitido el exceso espiritual que vuelve el arte auténticamente expresivo. La arquitectura no es, tradicionalmente, sino la fijación de una materia pesada sometida a las leyes de la gravedad, no superando por ello los límites de una expresividad puramente exterior y cosificada.

Por suerte, el prejuicio hegeliano ha sido ampliamente puesto en tela de juicio. Un edificio no es solo masa petrificada, ya que por arquitectura se entiende por supuesto otra cosa: ya no el arte vuelto primariamente a la constitución y mantención de un espacio interno que se erige frente al espacio mundano (según el modelo del edificio, de la casa, de la catedral), sino que se ha hecho dominante en las consideraciones filosóficas de la arquitectura (desde Le Corbusier (1998) a lo menos $^{1}$ - por lo menos en las intenciones-) la idea de que la arquitectura sea primitivamente el lugar de creación de esta misma división (interno/externo). Es decir que la arquitectura se sustenta primariamente en la fuerza que instituye en el espacio la diferencia dentro/fuera: ella es lo que da lugar, más que a lo estable, a la división y, por ello, al movimiento de entrada y a la posibilidad de una evasión del espacio. La "cosa arquitectónica" es algo, en este sentido, que no se repliega en sí, que no se cierra, y que, al contrario, describe primariamente líneas de fuga.

Esto vale para todos los tiempos y todas las construcciones, desde las pirámides, y bien antes en rigor: la arquitectura no es, o no es únicamente, masa espacial, sino que tiene principalmente que ver con el movimiento que excede la solidez de la materia y procede desde la fluidez del pasar o del huir. Lo arquitectónico tiene lugar como lo que, en el espacio, descubre el espacio; es decir, como lo que, formando lugares, teniendo lugar, produce sentido, o sea, abre los lugares en la medida en que los vuelve atravesables y evadibles. Se habla aquí de "evasión" en un sentido muy amplio y muy genérico, aludiendo a todo movimiento vuelto a salir del encierro, a todo gesto que deshace la conformación inerte del cuerpo a su ambiente, es decir, a toda resistencia a la clausura, a toda resistencia a la neutralización del cuerpo en una tensión definida o en una pose. $\mathrm{La}$ evasión sería, por consecuente, lo que implica "una necesidad de excedencia", como dijo Lévinas, y "la necesidad de salir de uno mismo, es decir, de romper el encadenamiento más radical, más irremisible, el hecho de que el yo es uno mismo" (Lévinas 82-3).

${ }^{1}$ Cf. para citar algunos trabajos filosóficos recientes que van en la misma línea se reenvía a: Deguy 1987, Nancy 1999, Goetz 2001, Radkovski 2002, Gaudin 2003, Gaudin y Mühlethaler 2005. 


\section{Del monumento a la "muralidad"}

En este sentido, es decisivo romper el hábito que nos hace asociar la arquitectura al edificio, a la estructura cerrada, a la 'habitación' o al 'domicilio', como este espacio en el que, como dijo Humberto Giannini en La reflexión cotidiana, "cumplo el acto más simple y real de un regreso a mí mismo, o más a fondo todavía, de un regressus ad uterum" (32).

La espacialidad enfatizada por Giannini no es sino una sedimentación pasiva y olvidadiza del primitivo traspasar y franquear, o sea, de las fuerzas que abren el espacio. Así, en antítesis con esta visión, la arquitectura debe pensarse a partir, por así decir, de la "muralidad", esto es, de la fuerza instituyente de muros y divisiones que, en lugar de encerrar en sí o en el uterum, rompe la totalidad del espacio dado y fractura lo ilimitado. Este sería en un sentido su primer impulso: la arquitectura corta, incurva, encuadra, fractura, escinde $y$, en todo ello, primariamente, desvía. Hace acontecer la fluidez del movimiento (del desvío).

Como afirma en 1924 Le Corbusier, la arquitectura: construye muros, muros que se extienden y se enlazan para ensancharse aún más. De este modo crean volúmenes, base de la sensación arquitectónica, sensación sensorial (150).

El muro (y no la casa) sería en este sentido el lenguaje primitivo de la arquitectura. El volumen o el espacio cerrado no serían nada más que un efecto derivado y secundario de la "muralidad", la cual implica más bien la construcción de tránsitos, de pasajes, de brechas a través de las cuales se pasa o no se pasa (es el pasar que está en juego y no el quedarse). Aquí la arquitectura es entonces ideación de pasantes, institución de la movilidad, que habría que oponer a toda idea de una arquitectura del hábitat o del habitante o del residente - del encierre del chez soi, o del uterum, justamente (a no ser que lo consideremos únicamente en el acto de nacer, de 'dar a luz', como se dice, que no es evidentemente sino una salida del encierre).

\section{La materia de la arquitectura}

Es, por cierto, digno de nota el hecho que la arquitectura no da forma a una materia que le preexiste, como en el caso de la escultura, que solo plasma la piedra; la arquitectura dispone, abre, agencia los espacios, mueve los caminos, no plasmando otra cosa que el 'espacio vacío'. En esto, el aparato comprensivo de Hegel se habría quedado corto: la arquitectura es una forma de arte muchos menos "material" de lo que se ha imaginado, muchos menos cercana al objeto "habitación" o "edificio" o "monumento" en tanto cosas físicas y estables y mucho más próxima a una "fuerza instituyente". 
Es precisamente en este sentido que Deleuze y Guattari en ¿Qué es la filosofía? oponen a la arquitectura-objeto o al monumento, la arquitectura-potencia o el arte del espaciamiento:

Es cierto que cada obra de arte es un monumento, pero el monumento no es aquí lo que conmemora un pasado, es un bloco de sensaciones presentes que no deben sino a ellas mismas su propia conservación, y dan al acontecimiento el compuesto que lo celebra (154).

Así, una obra-monumento no es simplemente lo que tiene lugar y que reposa y perdura en su ser-logrado, porque en rigor, detrás de la monumentalidad del monumento, yace la fuerza primitiva de su institución (el "bloco de sensaciones presentes") que emerge en una eterna repetición y que transciende completamente la estabilidad de su sentido acabado. Más allá de la obra-monumento, más allá del objeto muerto y de la obra de arte como objeto funerario (o sea, meramente conmemorativo), está la experiencia del acontecimiento arquitectural en tanto creación de espacio.

\section{El material arquitectural}

Esta observación altera en profundidad la mirada hacia la arquitectura en su conjunto: de modo no diferente a la danza o a una coreografía (volveré sobre esta analogía más adelante, pero volveré precisamente con el fin de que no se la vea únicamente como una analogía), la arquitectura-potencia se vislumbra como una escritura del movimiento de los cuerpos que interviene en el espacio para segmentarlo y abrir sus intersticios de fuga. Antes de darse como expresión de un proyecto, esto es, del sentido saturado de un volumen cerrado, o de exhibirse como pretensión de control, de captura, de bloqueo, o antes siquiera de ejecutar una mirada unitaria sobre el espacio, la arquitectura vendría haciendo visibles las fuerzas no-visibles que abren el espacio a sus posibles.

Debemos entonces corregir algo que dijimos: hemos afirmado que la arquitectura "plasma el espacio vacío", pero el verbo plasmar podría hacer creer que el espacio sea la "materia" de la arquitectura; sin embargo, no es que la arquitectura intervenga en aquel recortándolo, como el escultor interviene en la piedra descartando lo que le sobra para ser obra, porque al contrario hay que considerar que el recorte del espacio no es un acontecimiento que ocurre en el espacio o al espacio, sino que es el espacio mismo. El espacio no existe sino en la disyunción de los lugares, en la tensión de lo que no cesa de dividirse y de recortarse. Es la infinitud que no para de darse figuras finitas, definiendo los lugares con sistemas de relaciones: adentro/afuera, abierto/cerrado, pequeño/grande, alto/bajo, adelante/atrás, derecha/izquierda. Sencillamente el 
espacio no existe fuera de estas relaciones, no existe antes o fuera de los recortes. El espacio arquitectural coincide así con la dimensión aplicativa primordial del recortar: es la apertura a la infinitud de la variación espacial que crea nuevos lugares finitos, esto es, espacios sensatos, apropiados por los sentidos del cuerpo y por el gesto. El espacio es lo que da paso a los lugares, saturándose cada vez, pero excediendo cada vez toda saturación finita ya que es de suyo generador de nuevos gestos.

\section{La línea de fuga}

Para decirlo ahora con el léxico de Deleuze, edificar implica un movimiento que vectorializa, esto es, que divide el espacio en perspectivas, distancias, gestos, y los recompone según líneas de fuga.

Hay tres diferentes líneas que nos componen, según Deleuze (Deleuze y Parnet 142-152). Hay "líneas a segmentos duros": son las líneas más visibles que constituyen al hombre en segmentos definidos (la familia, la escuela, el sexo, la profesión, la clase social), en las que simplemente nos declinamos binariamente en un segmento o en otro - un hombre no es una mujer, un adulto no es un niño, un trabajador no es un estudiante, un juez no es un contable, etc. - : estas líneas son aquellas que definen los dispositivos de poder que trabajan en nuestro cuerpo, en nuestros deseos, en nuestros pensamientos. Hay enseguida "líneas flexibles" que modifican o generan desequilibrio en el plano, pero no producen sino microdevenires que no pasan el umbral de la visibilidad, y que sin embargo detallan el modo de nuestras relaciones: es el nivel de los secretos y de la existencia no manifiesta, de los desvíos subversivos en relación con el orden oficial en que vivimos, pero que no dan sino pequeñas fisuras imperceptibles arrastradas a los segmentos del poder establecido, que a veces no hacen sino confirmarlo. Y el tercer tipo de líneas, son precisamente las líneas que nos conciernen aquí, las "líneas de fuga": son aquellas que introducen una dimensión imprevisible y desconocida, procediendo a una verdadera ruptura con todo lo conocido y lo dado: no modifican los segmentos dados, sino que generan nuevos territorios.

La línea de fuga es en Deleuze un principio de variación infinita, es el principio generador del "dejar huir", o sea, la praxis de generar una nueva distribución, un nuevo agenciamiento de los posibles. La línea de fuga es el trazo que se realiza cuando se logra quedar atrapado en un devenir, que no es, por supuesto, ni un desarrollo ni una evolución. Es una verdadera revolución, o sea no es un enfrentamiento reactivo con respecto al orden establecido, sino que es una fuerza afirmativa completamente nueva, que escapa a la centralización y huye a la totalización y que, sobre todo, es capaz de rediseñar enteramente el sentido de las relaciones segmentadas, alterando su funcionamiento. 
Deleuze alude a esto en los Diálogos con Claire Parnet con una imagen muy eficaz:

Habría que ser como un taxi: línea de espera, línea de fuga, embotellamiento, estrechamiento de calzada, semáforos en verde y en rojo, ligera paranoia, complicaciones con la policía. Una línea abstracta y quebrada, un zig-zag que se desliza «entre» (Deleuze y Parnet 38).

La línea de fuga es la que trazo cuando busco huir al bloqueo del tránsito, la cual se ofrece en la desorganización de lo configurado anteriormente: el taxi en el taco reinventa el movimiento, se desliza en los intersticios, asume y desvía la paranoia espacial. No procede por axiomas o por geometrías abstractas, no instituye un territorio cerrado, sino que reinventa el espacio, descubre trayectorias antes inimaginables: en otras palabras, juega con una espacialidad en tanto acontecimiento, o capaz de exponerse al acontecimiento.

Ahora, esta reinvención del espacio es primariamente una deterritorialización. Puede leerse esta conexión en los mismos Diálogos:

La línea de fuga es una desterritorialización. Los franceses no saben muy bien lo que es eso. Por supuesto, como todo el mundo, huyen, pero piensan que huir, o bien es escaparse del mundo, mística o arte, o bien es una especie de cobardía, una manera de eludir los compromisos y las responsabilidades. Pero huir no significa, ni muchísimo menos, renunciar a la acción, no hay nada más activo que una huida. Huir es lo contrario de lo imaginario. Huir es hacer huir, no necesariamente a los demás, sino hacer que algo huya, hacer huir un sistema como se agujerea un tubo. [...] Huir es trazar una línea, líneas, toda una cartografía. Sólo hay una manera de descubrir mundos: a través de una larga fuga quebrada (Deleuze y Parnet 45).

Si se quiere abrir mundos espaciales, se trata de hacer huir o de hacer que algo huya, de quedarse atrapado por el devenir, esto es, de pervertir lo espacial dado fisurándolo con el movimiento lineal. Trazar una línea de fuga en un plan es dar otro punto de vista sobre el conjunto de una situación espacial, es derivar un criterio inmanente que permite generar agenciamientos que se regeneran, se desterritorializan, se estratifican, abriendo a nuevos agenciamientos y a un nuevo devenir determinado espacialmente.

\section{Fuerzas danzantes}

Así, un mundo arquitectural sería aquel completamente transformado en acontecimiento espacial, desvinculado de la permanencia del monumento. La dimensión monumental de la arquitectura, de los edificios, puede ser eficaz en términos estéticos, o en términos simbólicos (como el útero del que habla 
Giannini), pero no es sino el punto de partida para volver a plantear el problema de la creación del espacio: nunca el espacio es un "adentro" pleno, nunca solo una ergonomía desplegada; el espacio se describe a partir de intersecciones, entrelazos, tensiones de fuerzas, desorientaciones que tienden a reajustar el oriente. Cuando un espacio adquiere arquitectónicamente sentido, lo hace permitiendo signos, gestos, movimientos del cuerpo que, en cierto modo, escapan al mero control.

Ahora bien, este campo que estamos describiendo es aquel de la arquitectura e igualmente aquel de todas las otras formas de creación artística que solo encuentran su sentido en la búsqueda de superar los límites de lo dado. Todos los artistas son, para Deleuze, exploradores de territorios desconocidos de la experiencia. Pero son los bailarines en particular aquellos que se mueven en las profundidades corpóreas del lugar naciente del sentido (el cuerpo) y que al mismo tiempo acompañan esta bajada por un contramovimiento de recuperación en la superficie, lo que evita que la danza se convierta en un mero abandono al afecto del cuerpo. La danza es la repetición en la superficie de las profundidades del cuerpo. Es decir, es escritura del cuerpo en movimiento. En esta experiencia límite el bailarín libera ya no un delirio privado o una mera "línea flexible", como si se tratara de una alucinación solitaria y privada. El bailarín -que, tal como en general los artistas, bordea el abismo del sentido corriendo incluso el riesgo de quedarse atrapado en esta alucinación, como sucede en muchos artistas que Deleuze cita: Fitzgerald, Bacon, Kleist- es aquel que excede el mero juego de la flexión, esto es, de la oposición al sistema de sentido configurado, ya que más que deshacer los movimientos dados, abre brechas "de fuga", abre paisajes completamente nuevos para el movimiento. Bailar es trazar líneas en el espacio que antes eran inexistentes $\mathrm{y}$, con ello, formar dinámicas y figuras en movimiento que son al mismo tiempo presentes y evanescentes.

El bailarín, dice Deleuze en La imagen-tiempo -casi interpretando a su manera o extendiendo el ejemplo de Lyotard que citamos al comienzo-, es "un sonámbulo que será poseído por el movimiento que parece" (1987 88).

Ser poseído por el movimiento o por el espacio de tal modo que es el movimiento o el espacio aquel que llama, implica no solo repetir mecánicamente y económicamente un movimiento (como en el ejemplo del somnámbulo de Lyotard), sino escapar a los movimientos codificados y ritualizados de los segmentos duros, esto es, permitir una metamorfosis del cuerpo que rompe todas las oposiciones binarias. Se transgrede con ello la identidad dominada del cuerpo sometido al control o al autocontrol, lo que permite difuminar la representación de sí en la que el cuerpo tiene una asignación fija. Esta huida a través del arte deshace el sujeto, explora las regiones de lo insignificante, de lo que Deleuze llama "devenir animal". La danza forma extraños y nuevos devenires: deja libre el espacio para que los rasgos de esta animalidad aparezcan, hasta crear una zona de indiscernibilidad entre hombre y 
animal en lo que Deleuze llama “carne” (1993 14). Ahí se anuda la analogía entre arquitectura y danza que desemboca incluso en una indiferenciación: deberíamos pensar que hay arquitectura y danza -que hay arquitectura, esto es, danza-, allí donde el espacio se vuelve "animal", o sea, allí donde se permite una desorientación o una reorientación del movimiento y donde se desconfigura el espacio para abrir nuevos dinamismos de inmanencia a un "devenir arquitectuanimal".

\section{Bibliografía}

Deguy, Michel. “La vrai ville et absente”. Les temps de la réflexion, 8 (1987): 273-286.

Deleuze, Gilles. La imagen-tiempo. Estudios sobre cine 2, trad. I. Agoff. Barcelona: Paidós, 1987.

Deleuze, Gilles y Guattari, Félix. Qu'est-ce que la philosophie? Paris: Editions de Minuit, 1991.

Deleuze, Gilles. Francis Bacon. Lógica de la sensación. Barcelona: Arena, 1993.

Deleuze, Gilles y Parnet, Claire. Diálogos, trad. J. Vázquez. Valencia: Pre-textos, 1980.

Gaudin, Christian. Considérations sur l'espace. Paris : Rocher, 2003.

Gaudin, Christian y Mühlethaler, Laure. Édifier: l'architecture et le lieu. Paris : Flammarion, 2005.

Giannini, Humberto. La reflexión cotidiana. Santiago: Universitaria, 1987.

Goetz, Benoît. La dislocation. Architecture et philosophie. Paris: Passion, 2001.

Goetz, Benoît. Théorie des maisons. L'habitation, la surprise. Lagrasse: Verdier, 2011.

Hegel, Georg Wilhelm Friedrich. La arquitectura, trad. A, Clavería. Barcelona: Kairós, 1981.

Le Corbusier. Hacia una arquitectura, trad. J. Martínez Alinari. Barcelona: Apóstrofe, 1998.

Lévinas, Emmanuel. De la evasión, trad. J. Rolland. Madrid: Arena, 1999.

Lyotard, Jean-François. Misère de la philosophie. Paris: Galilée, 2000

Nancy, Jean-Luc. La ville au loin. Paris: Mille et une nuits, 1999

Nietzsche, Friedrich. La gaya ciencia, Obras completas 3, trad. J. Aspiunza y otros. Madrid: Tecnos, 2014.

Radkovski, Georges. Anthropologie de l'habiter: vers le nomadisme. Paris : Puf, 2002.

Wittgenstein, Ludwig. Aforismos. Cultura y valor, trad. E. Frosi. Madrid: Espasa Calpe, 1995. 